\title{
Theoretical investigation of redox species in condensed phase
}

\author{
NITAL MEHTA and SAMBHU N DATTA* \\ Department of Chemistry, Indian Institute of Technology-Bombay, Powai, Mumbai 400076 \\ e-mail: sndatta@chem.iitb.ac.in
}

MS received 4 June 2007; accepted 7 August 2007

\begin{abstract}
We give a detailed description of the use of explicit as well as implicit solvation treatments to compute the reduction potentials of biomolecules in a medium. The explicit solvent method involves quantum mechanical/molecular mechanics (QM/MM) treatment of the solvated moiety followed by a Monte-Carlo (MC) simulation of the primary solvent layer. The QM task for considerably large biomolecules is normally carried out by density functional treatment (DFT) along with the MM-assisted evaluation of the most stable configuration for the primary layer and biomolecule complex. The MC simulation accounts for the dynamics of the associated solvent molecules. Contributions of the solvent molecules of the bulk towards the absolute free energy change of the reductive process are incorporated in terms of the Born energy of ion-dielectric interaction, the Onsager energy of dipole-dielectric interaction and the Debye-Hückel energy of ion-ionic cloud interaction. In the implicit solvent treatment, one employs the polarizable continuum model (PCM). Thus the contribution of all the solvent molecules towards the free energy change are incorporated by considering the whole solvent as a dielectric continuum.

As an example, the QM(DFT)/MM/MC-Born/Onsager/Debye-Hückel corrections yielded the oneelectron reduction potential of Pheophytin- $a$ in the solvent DMF as $-0.92 \pm 0.27 \mathrm{~V}$ and the two-electron reduction potential as $-1.34 \pm 0.25 \mathrm{~V}$ at $298.15 \mathrm{~K}$ while the DFT-DPCM method yielded the corresponding values as $-1.03 \pm 0.17 \mathrm{~V}$ and $-1.30 \pm 0.17 \mathrm{~V}$, respectively. The calculated values more or less agree with the observed mid-point potentials of $-0.90 \mathrm{~V}$ and $-1.25 \mathrm{~V}$, respectively. Moreover, a numerical finite difference Poisson-Boltzmann solution along with the DFT-DPCM methodology was employed to calculate the reduction potential of Pheophytin- $a$ within the thylakoid membrane. The calculated reduction potential value of $-0.58 \mathrm{~V}$ is in agreement with the reported value of $-0.61 \mathrm{~V}$ that appears in the socalled $Z$-scheme and is considerably different from the value in vitro.
\end{abstract}

Keywords. Biomolecules; reduction potential; QM/MM/MC; PCM.

\section{Introduction}

In recent years, an extensive amount of experimental and theoretical research has been carried out on the electron transport pathway involved in the light phase of photosynthesis in green plants. ${ }^{1-4}$ The results of these investigations have given rise to a clear picture of the arrangement of molecules within the thylakoid membrane and of the solvent molecules associated with a species in solution. Newton et al investigated electron transfer reactions in condensed phases. ${ }^{5}$ Parson and Warshel prepared a density matrix model of photosynthetic electron transfer with microscopically-based estimates of vibrational relaxation times. ${ }^{6}$ Our activity in this field has involved the study of the thermodynamical and kinetic properties of biomolecules involved in the $Z$-scheme of photosynthesis. ${ }^{7}$

\footnotetext{
*For correspondence
}

The thermodynamical work consists of theoretical calculation of the reduction potentials of various biomolecules in vivo and in vitro, while the kinetic study has been on the calculation of the rate of electron transfer between two biomolecules within the semirigid condensed phase of the thylakoid membrane. ${ }^{8}$

This paper gives a general description of the quantum chemical methodology we employ while determining the reduction potential of a general molecule in a specific medium. A number of energy terms, such as the electronic energy, different contributions to the solvation energy, and thermal energy, are required to be evaluated here. Calculation of the molecule-medium interaction energy takes the central stage and is discussed in detail. The molecular geometry is influenced by the conformation of the solvents in its immediate vicinity, and vice-versa. Also, the solvent molecules are always moving relative to the solute molecule. So, the basic task is to make an average over 
the various lowest-energy solute-associated solvent conformations. This can be broken down in three distinct steps: (1) evaluation of the most preferred solute geometry in the presence of static solvent molecules; (2) equilibration of the dynamic solvent molecules starting from the configuration in the first step; and (3) averaging over different solvent conformations starting from the equilibrium one. As an example, we show how the one-electron and two-electron reduction potentials of Pheophytin- $a$ in the solvent $N, N$-dimethyl formamide (DMF) can be theoretically determined and its one-electron reduction potential within the thylakoid membrane can be effectively estimated. ${ }^{9}$

\section{Methodology}

The basic task is to compute the difference of electronic, thermal and solvation energies of the anionic form of a molecule from that of its neutral form in the presence of a polarizable medium. The calculated absolute free energy $(G)$ of these species can be used to obtain the absolute free energy change $(\Delta G)$ of the reductive process. In turn, the mid-point potential is obtained from the calculated absolute free energy change and the absolute free energy associated with the reduction involved in the hydrogen electrode. The latter quantity has been reliably determined by Cramer et al. ${ }^{10}$

\subsection{Geometry optimization}

First of all, it is necessary to optimize the geometry of the molecule in presence of the molecules of the medium (solvent molecules). This can be accomplished in two ways. First, one may consider the solvent molecules explicitly via a quantum mechanics molecular mechanics - Monte-Carlo (QM/MM/MC) approach. In this elegant procedure, the solute is described quantum mechanically, its interaction with the solvent molecules assessed by molecular mechanics, and finally the Monte-Carlo procedure accounts for the dynamics of the solvent molecules. The second and the easier way is to visualize the solvent implicitly as a dielectric continuum, and adopt a polarized continuum model (PCM). Gaussian 03 (G03) suite of programs ${ }^{11}$ can be used in all the calculations.

We stress here that for an accurate evaluation of the energy difference, the geometry of each species (neutral and ionic forms) must be separately optimized, and the optimization process must include the effect of the surrounding molecules of the medium.

\subsection{Electronic energy}

In the $\mathrm{QM} / \mathrm{MM} / \mathrm{MC}$ method, the optimized structures of neutral and the charged forms of the solute molecule are obtained by the ONIOM method ${ }^{12}$ by restricted open-shell density functional treatment (DFT) such as at ROB3LYP (restricted open shell Becke 3-parameter Lee, Yang and Parr exchange correlation functional) level using a medium size basis set like $6-31 \mathrm{G}(\mathrm{d})$, while the solvent molecules are treated by the universal force field (UFF) ${ }^{13}$ Electronic energies of the neutral and charged species are finally obtained by carrying out single point calculations on the ONIOM optimized geometry at the ROB3LYP level using a much larger basis such as 6-311 + G (2d, 2p:UFF).

In the DFT-DPCM (Dielectric Polarizable Continuum Model) method, electronic energies are obtained by optimizing the geometry of the neutral and charged species at ROB3LYP level using a smaller basis and then by single point PCM calculations at the same level but using the larger basis set.

\subsection{Thermal energy and molecular entropy}

Thermal energy and entropy contribution towards the free energy change of the reductive process are obtained for the optimized geometry of a truncated model of the neutral or charged species. This is because of two reasons. The first one is operational. Most of the quantum chemical softwares presently allow thermal energy calculations based on normal mode analysis for a rather small molecule, such as one with not more than 50 atoms. The second reason involves logistics. Each thermal energy calculation with a good enough basis set takes a long computing time, of the order of thousands of cpu hours. A truncated model is necessary to bring the computing time down to about $200 \mathrm{cpu}$ hours. The model, however, must be carefully chosen. For porphyrin-based molecules, we have always found that while the calculated difference in electronic energy is very sensitive to the substituents, ${ }^{7 \mathrm{~g}}$ the calculated thermal energy difference, generally a small quantity in electron Volt, remains more or less unchanged upon replacement of most of the substituents by hydrogen atoms. ${ }^{7 \mathrm{~g}}$ Thus the basic porphyrin skeleton provides a good estimate of the thermal energy difference between the neutral and anionic forms of molecules like chlorophyll- $a$, pheophytin- $a$, etc. Nevertheless, the molecular geometry of each model system with different 
charges must be separately optimized at first, and the optimized geometries should be used for normal mode frequency analysis.

\subsection{Solvation energy}

We employ both the explicit (QM/MM/MC + Born/Onsager/Debye-Hückel) model and implicit solvation model (DFT-DPCM) to account for the contribution of the solvent molecules to the free energy change of the reduction process.

In the QM/MM/MC model, the primary solvent shell of about 50 solvent molecules is treated. A constant temperature $(298.15 \mathrm{~K})$, constant volume (cubic unit cell of volume of about $25 \times 25 \times 25 \AA^{3}$ ) (NVT) ensemble is adopted. Configurations of the solvent molecules around the neutral and ionic species are initially optimized under the molecular mechanics (CHARMM27) force field by employing the Steepest Descent algorithm. The optimized configuration is allowed to relax at a regular max delta (maximum trial atomic displacement) of $0.05 \AA$ and for a total of 1000 run steps to simulate an adequate description of the MC solvent dynamics. The system is equilibrated for the first 300 run steps. Thereafter, data are collected after each run step. The remaining 700 data points are averaged by Boltzmann distribution. The standard deviation of the simulated energies is calculated for each of the neutral and anionic species. To reduce the finite boundary effects and to mimic the infinite system, periodic boundary condition is applied. To neutralize the charges of the anion and dianion, one and two $\mathrm{Na}^{+}$ions are introduced at the centre of the cubic box. Mulliken charges generated by ROB3LYP calculations using the 6-31G $(d)$ basis sets for the neutral and ionic forms of the solute molecule and for the neutral solvent molecule are used. Hyperchem Professional Release 7 for Windows ${ }^{14}$ can be used in these calculations.

\subsection{Perturbative corrections to solvation energy}

The remaining solvent is treated as a structureless dielectric continuum.

The Born free energy of the ion-dielectric interaction is given by

$$
G_{\text {Born }}=-\frac{Q^{2}}{2 a_{0}}\left(1-\frac{1}{\varepsilon}\right)
$$

where $a_{0}$ is the radius and $Q$ is the total charge of the solute-primary-solvation-layer complex. The quantity $\varepsilon$ is the dielectric constant of the bulk solvent.

The Onsager free energy of the dipole-dielectric interaction is given by

$$
G_{\text {Onsager }}=-\frac{(\varepsilon-1)}{(2 \varepsilon+1)} \frac{\mu^{2}}{a_{0}^{3}}
$$

where $\mu$ is the ground state dipole moment of the solute-layer complex.

Gaussian 03 is used to obtain the values of $a_{0}$ and $\mu$ of the neutral and anionic forms of the solvated species at ROB3LYP/6-31G(d) level.

The additional stabilization provided by the medium through the ion-ionic atmosphere interaction is estimated by using Debye-Hückel theory. We write

$$
G_{D H}=-\frac{Q^{2} \kappa}{2 \varepsilon}
$$

The Debye-Hückel reciprocal length $\kappa$ is given by

$$
\kappa=\left[\frac{4 \pi e_{0}^{2}}{\varepsilon k_{B} T} \sum_{i}\left(\frac{N c_{i}}{1000}\right) z_{i}^{2}\right]^{1 / 2}
$$

where $e_{0}$ is the electronic charge, $k_{B}$ is the Boltzmann constant, $T$ is the temperature of the system in Kelvin, $N$ is the Avogadro number, $c_{i}$ is the concentration of the $i$ th species in molar unit, and $z_{i} e_{0}$ is the charge of the $i$ th ion. ${ }^{15}$ We take $1 \mathrm{M}$ concentration for each type of the ions (with the corresponding ionic strength $1 \mathrm{M}$ for anionic solution and $4 \mathrm{M}$ for dianionic solution).

For a specific process in a solvent medium,

$$
\begin{aligned}
& \Delta G_{\text {red }}^{o}=\Delta(E)_{\text {model }, Q M}+\Delta E_{\text {thermal }} \\
& +\left(\Delta G_{M C}+\Delta G_{B o r n}+\Delta G_{\text {Onsager }}+\Delta G_{D H}\right)-T \Delta S
\end{aligned}
$$

while neglecting the $P V$ contribution as it is negligibly small for solvated species.

\subsection{Alternative calculation of medium interaction energy}

We also employ the DFT-DPCM methodology to obtain the free energy of reduction of a biomolecule in solution and within a condensed phase such as a membrane. The finite difference Poisson-Boltzmann (FDPB) method ${ }^{16,17}$ along with the DPCM solvation 
model at ROB3LYP/6-31G(d) level are normally employed to obtain the total free energy in a membrane. The electrostatic and non-electrostatic contributions to the free energy change during the reduction process are obtained by employing the FDPB and DPCM methods respectively.

We then obtain

$$
\Delta G_{\text {red }}^{o}=\Delta G_{e l}+\Delta G_{n o n-e l} .
$$

\section{Standard reduction potential}

The standard reduction potential is written as

$$
\begin{aligned}
E_{r e d}^{o}(\text { in } V)=- & \frac{1}{n_{e}}\left[\Delta G_{r e d}^{o}(\text { in } e V)\right. \\
& \left.-\Delta G_{H^{+} /(1 / 2) H_{2}(\mathrm{~g})}^{o}(\text { in } e V)\right],
\end{aligned}
$$

where $n_{e}$ is the number of electrons transferred in the half-cell reaction and the $\Delta G^{o}$ values are quantities per molecule. We take $\Delta G_{\left[H^{+}(\mathrm{aq}) \rightarrow(1 / 2) \mathrm{H}_{2}(g)\right]}^{o}=$ $-4.36 \mathrm{eV}$ from ref. 10.

\section{Application}

The methodologies described above were successfully used to calculate the absolute free energy of reduction of Plastocyanin in water ${ }^{7 \mathrm{~g}}$ Chlorophyll- $a$ in acetonitrile, ${ }^{7 \mathrm{~h}}$ and Pheophytin- $a$ (Pheo- $a$ ) in solvent DMF and also in thylakoid membrane. ${ }^{9}$ In the following, we discuss our most recent work on Pheophytin- $a$.

Pheophytin- $a$ (Pheo- $a$ ) is one of the biomolecules involved in the electron transfer process in the light phase of photosynthesis in green plants. The choice of the molecular geometry is discussed in the following.

There are 141 atoms in Pheo- $a$ as shown in figure 1. For computational purposes, we worked on the truncated forms Pheo-47 and Pheo-86, species with 47 and 86 atoms respectively. The optimized geometry of the bare neutral species are shown in figures 2(a) and (b) respectively. Pheo-47 has the vinyl group intact and all the other side chains replaced by hydrogen atoms. Pheo-86 retains all the side chains intact except that the phytyl chain is replaced by an ethyl group.

The thermal energy and the molecular entropy were obtained from a harmonic analysis of the vibrational frequencies. Frequency calculations were done on bare Pheo-47, its anion and di-anion, each optimized at ROB3LYP/6-31G(d) level. The adoption of the truncated model is justified because the additional charge in the anion and dianion is localized largely within the $\pi$ frame, and consequently the bond force constants for the side chains do not change significantly on ionization. Moreover, the difference in thermal energy between the neutral and charged species contributes negligibly towards the change in free energy during the process of reduction.

For the ONIOM and PCM calculations on Pheo- $a$ in solvent DMF, we started from the vacuum-optimized geometries of Pheo-86 at ROB3LYP/6-31G(d) level

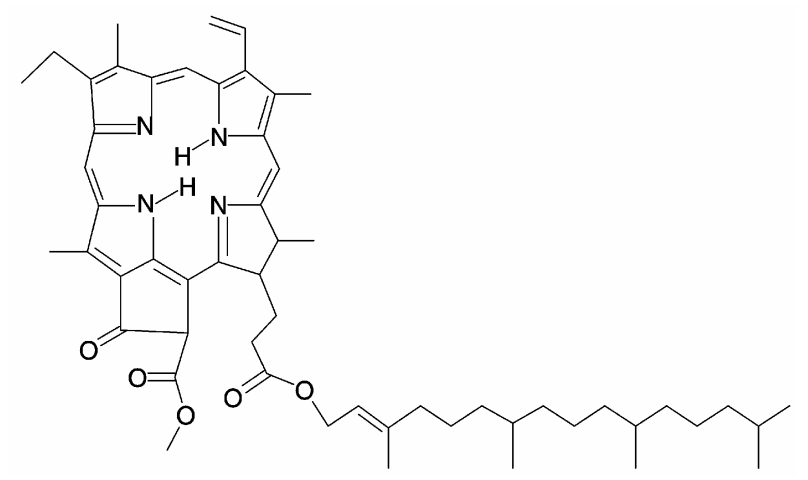

Figure 1. Structure of Pheophytin- $a$ (reproduced with permission from (J. Phys. Chem. B, in press). Copyright 2007 American Chemical Society).
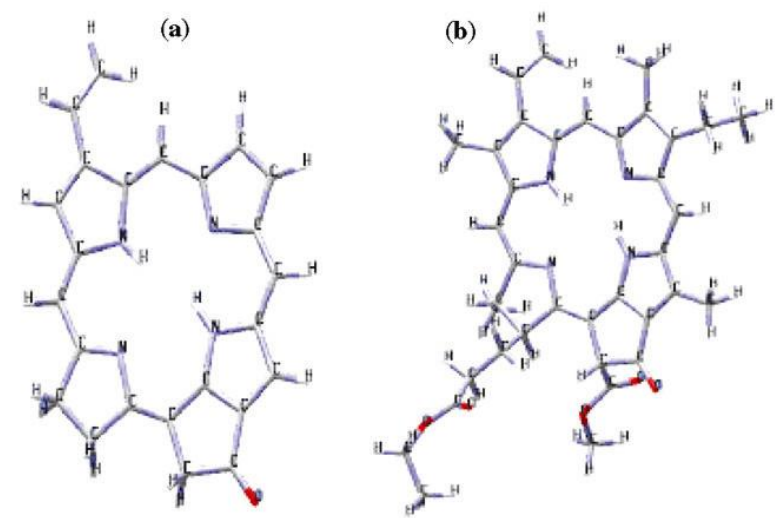

Figure 2. Gas phase optimized geometry of the truncated models of Pheophytin- $a$ : (a) Pheo-47 with all substituents except the vinyl group replaced by hydrogen, and (b) ethyl derivative Pheo-86 with all the side chains retained except for the phytyl chain that is replaced by an ethyl group (reproduced with permission from (J. Phys. Chem. B, in press). Copyright 2007 American Chemical Society). 
and optimized the molecular geometries again in the presence of solvent. A snapshot of the ethyl derivative of Pheo-86 with the primary solvation shell of 45 DMF molecules is given in figure 3 . The geometry was optimized employing the ONIOM methodology at ROB3LYP/6-31G(d): UFF level. The primary solvation shell was further treated by $\mathrm{MC}$ simulation.

For the calculations on Pheo- $a$ in thylakoid membrane, a section of the overall structure of PSII was chosen from 2AXT entry in the Brookhaven Protein Data Bank. This section is shown in figure 4, and includes all the proteins and co-factors within a radial distance of $4 \AA$ from Pheo- $a$. It consists of the following residues: (a) 4 units of LEU, 4 ALA, 1 THR,

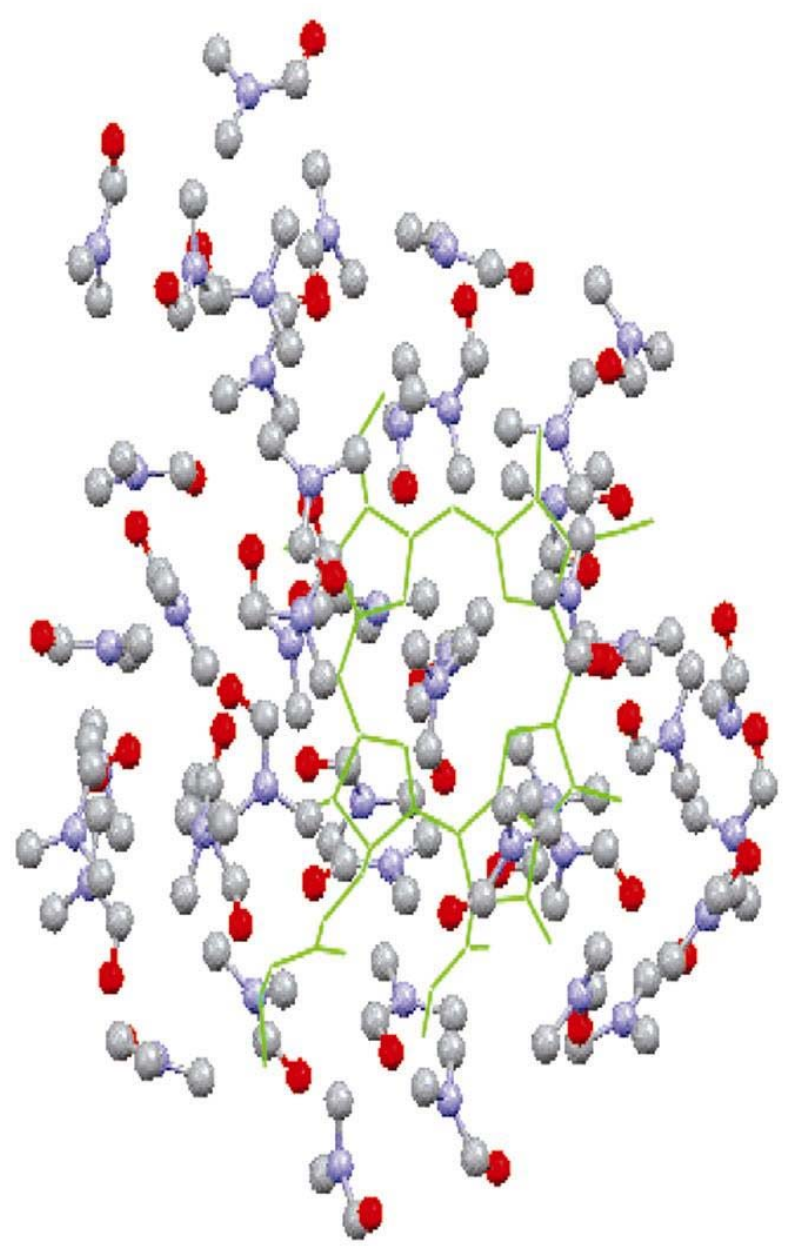

Figure 3. Pheo-86 with the primary solvation shell of $45 \mathrm{DMF}$ molecules. The primary solvation shell is shown by the ball and stick model. For the sake of clarity, hydrogen atoms are not shown here (reproduced with permission from ( $J$. Phys. Chem. B, in press). Copyright 2007 American Chemical Society).
2 ILE, 3 PHE, 2 TYR, 1 GLN, 2 PRO, 1 MET, 1 GLY, 1 VAL, 1 TRP; (b) 2 molecules of CLA (Chlorophyll-a), 1 MGE [(1S)-2-(alpha-L-allopyranosyloxy)1-[tridecanoyloxy)methyl]ethylpalmitate]; and (c) 1 PHO (Pheophytin- $a$ ). All atomic coordinates were taken from the crystal structure of Pheophytin- $a$ (PDB 2AXT). In the truncated model the cleaved peptide linkages were converted to free carboxylic $\left(\mathrm{COO}^{-}\right)$ or free amino $\left(\mathrm{NH}_{3}^{+}\right)$groups, as required. Both polar and non-polar hydrogen atoms were inserted into the proteins while only polar hydrogen atoms were inserted into the cofactors by the WHAT IF software ${ }^{18}$ and then their coordinates were optimized by the $\mathrm{MM}+$ force field.

\section{Results and discussions}

The softwares used for this kind of work are listed in table 1 . We used a value of 38.7 for $\varepsilon$ of DMF in our work.

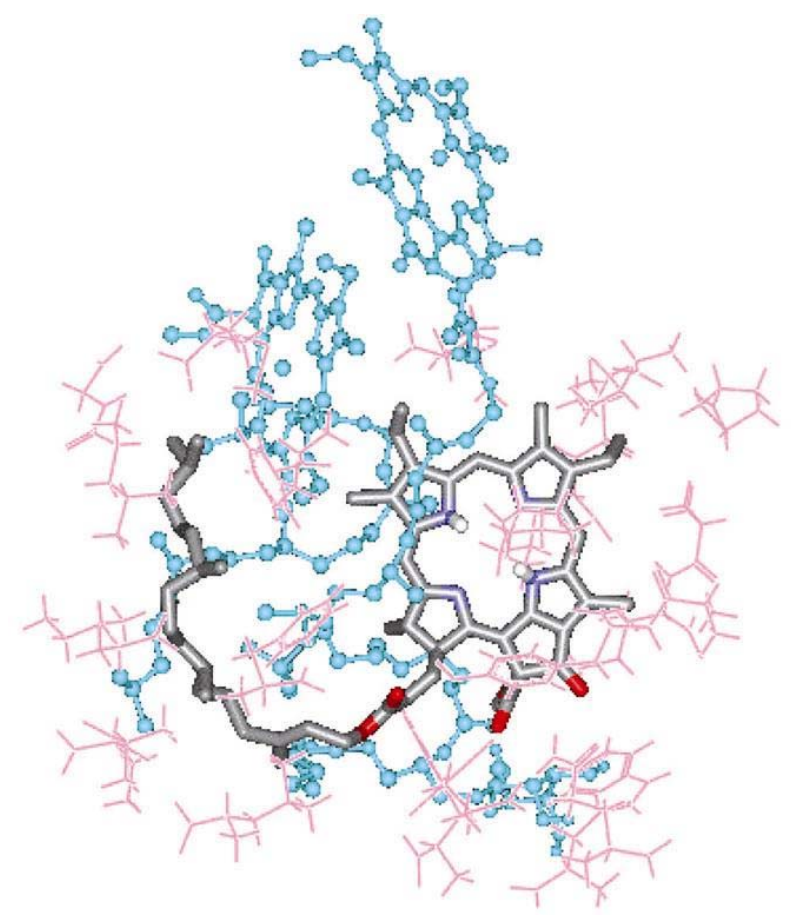

Figure 4. A section of the overall structure of PSII (from 2AXT entry in the Brookhaven Protein Data Bank). The truncated model consists of (a) 4 units of LEU, 4 ALA, 1 THR, 2 ILE, 3 PHE, 2 TYR, 1 GLN, 2 PRO, 1 MET, 1 GLY, 1 VAL, 1 TRP (shown as narrow sticks); (b) 2 molecules of CLA, 1 MGE (shown in ball and stick model); and (c) 1 PHO (shown as broad lines). (reproduced with permission from (J. Phys. Chem. B, in press). Copyright 2007 American Chemical Society). 
Table 1. Quantum chemical softwares necessary for investigation of a molecule in a medium.

\begin{tabular}{lll}
\hline Software & \multicolumn{1}{c}{ Relevant task } & Availability \\
\hline Gaussian 03 & $\begin{array}{l}\text { Geometry optimization, ROB3LYP calculation, } \\
\text { ONIOM technique, PCM computations. } \\
\text { GyperChem Professional } \\
\text { Release 7.01 }\end{array}$ & Purchase \\
$\begin{array}{l}\text { WHAT IF } \\
\text { Delphi }\end{array}$ & $\begin{array}{l}\text { Insertion of hydrogen atoms to the pdb file. } \\
\text { Calculation of electrostatic interactions in proteins. }\end{array}$ & Purchase \\
\hline
\end{tabular}

Table 2. Calculation of $\Delta H^{0}$ by QM/MM/MC method. ${ }^{9}$

\begin{tabular}{lcccc}
\hline & \multicolumn{4}{c}{$(\mathrm{eV})$} \\
\cline { 2 - 5 } Reaction & $\Delta(E)_{\text {model,QM }}$ & $\Delta E_{\text {thermal }}$ & $\Delta E_{\text {solv }}$ & $\Delta H^{0 \text { a }}$ \\
\hline Pheo(DMF) $+e^{-}(g) \rightarrow$ Pheo $^{-}(\mathrm{DMF})$ & -1.918 & -0.079 & $-1.415 \pm 0.266$ & $-3.412 \pm 0.266$ \\
Pheo(DMF) $+2 e^{-}(g) \rightarrow$ Pheo $^{2-}(\mathrm{DMF})$ & -0.890 & -0.158 & $-4.977 \pm 0.499$ & $-6.025 \pm 0.499$ \\
\hline${ }^{\mathrm{a}} \Delta H^{0}=\Delta(E)_{\text {model, } Q M}+\Delta E_{\text {thermal }}+\Delta E_{\text {solv }}$ & & &
\end{tabular}

To decide upon the number of solvent molecules to be used for the ONIOM calculations, we first employed 3-21G basis set and performed the ONIOM calculation on the neutral species. The molecular geometry of neutral Pheo-86 in 25, 50 and $100 \mathrm{DMF}$ molecules was optimized by employing the ONIOM (ROB3LYP/3-21G:UFF) methodology with mechanical embedding. For each total number of solvent molecules, the position of the solvent molecules in the ONIOM-optimized aggregate was slightly changed to obtain a new solvent conformation. The new conformation was then taken as the starting point for another ONIOM optimization, and the process was repeated several times. The energy of the active site $\left[E_{2}=(E)_{\text {model, QM }}\right]$ was noted. Three conformers were considered for 25 DMF molecules along with Pheo86 , six conformers were investigated for $50 \mathrm{DMF}$ molecules and one conformer was considered for $100 \mathrm{DMF}$ molecules. The energy difference $\left(E_{2}\right)_{\text {ONIOM }}-$ $E_{\text {bare }}$ oscillated widely when $25 \mathrm{DMF}$ molecules were considered. In the case of $50 \mathrm{DMF}$ molecules, the energy difference varied slightly around the value of 0.0028 a.u. The calculation involving $100 \mathrm{DMF}$ molecules led to more or less the same energy difference. Therefore, we settled for $45 \mathrm{DMF}$ molecules in all subsequent calculations. The reduction reactions considered are as follows:

$$
\begin{aligned}
& \text { Pheo }(\mathrm{DMF})+\mathrm{e}^{-}(\mathrm{g}) \rightarrow \text { Pheo }^{-}(\mathrm{DMF}) \\
& \text { Pheo }(\mathrm{DMF})+2 \mathrm{e}^{-}(\mathrm{g}) \rightarrow \text { Pheo }^{2-}(\mathrm{DMF}) .
\end{aligned}
$$

The calculation of $\Delta H^{0}$ is given in table 2. The energy of electron is nearly zero and neglected. Here $(E)_{\text {model, } Q M}$ is the energy of the pheophytin moiety at the QM level under the influence of 45 DMF molecules. It is the ONIOM single point energy at ROB3LYP/6-311+G(2d, 2p) level on the ONIOM (ROB3LYP/6-31G $(d)$ :UFF) optimized geometry. The quantity $E_{\text {thermal }}$ is the thermal energy for Pheo47 species at ROB3LYP/6-31G(d) level while $E_{\text {solv }}$ is the solvation energy for Pheo-86 species. Calculated error is the square root of the sum of squares of the individual standard deviations.

The $\Delta G_{r e d}^{0}$ calculated by QM/MM/MC+Born/ Onsager/Debye-Hückel method and the reduction potential calculated from (7) are given in table 3.

The $\Delta G_{\text {red }}^{0}$ calculated by DFT-DPCM method and the reduction potential calculated from (7) are given in table 4 where the error in the calculation of $\Delta G_{\text {red }}^{0}$ is the inherent uncertainty associated with the application of QM on large molecules and its magnitude is about $4 \mathrm{kcal} / \mathrm{mol}$.

In the FDPB method, all atomic coordinates were taken from the crystal structure (PDB 2AXT), cofactors and proteins within a radial distance of $4 \AA$ were considered, and radius was assigned to all the protein atoms from the Delphi ${ }^{19}$ database (CHARMM22 parameter set). The CHARMM22 parameter set was also employed to assign partial charges to all the atoms in the proteins, whereas the DFT electrostatic-potential (DFT-ESP) charges, that had been calculated with the keyword CHelpG at the ROB3LYP/6-31G(d) 
Table 3. Calculation of the absolute free energy of one- and two-electron reduction $\left(\Delta G_{r e d}^{0}\right)$ and $E_{\text {red }}^{0}$ of Pheophytin- $a$ in DMF by QM/MM/MC method. We have used 1 a.u. =27.21(16) $\mathrm{eV}$ and $1 \mathrm{eV}^{\mathrm{N}}=23.06(05) \mathrm{kcal} \mathrm{mol}^{-1}{ }^{9}{ }^{9}$

\begin{tabular}{|c|c|c|c|c|c|}
\hline \multirow[b]{2}{*}{ Reaction } & \multicolumn{3}{|c|}{$(e V)$} & \multicolumn{2}{|c|}{ Mid-point $E_{\text {red }}^{0}(\mathrm{~V})$} \\
\hline & $\Delta H^{0}$ & $-T \Delta S_{m o l}$ & $\Delta G_{r e d}^{0}{ }^{\mathrm{a}}$ & Calcd. & Obsd. \\
\hline Pheo(DMF) $+\mathrm{e}^{-}(\mathrm{g}) \rightarrow \mathrm{Pheo}^{-}(\mathrm{DMF})$ & $-3 \cdot 412 \pm 0 \cdot 266$ & $-0 \cdot 027$ & $-3 \cdot 440 \pm 0 \cdot 266$ & $-0 \cdot 920 \pm 0.266$ & $-0 \cdot 90$ \\
\hline Pheo(DMF) $+2 \mathrm{e}^{-}(\mathrm{g}) \rightarrow \mathrm{Pheo}^{2-}(\mathrm{DMF})$ & $-6 \cdot 025 \pm 0.499$ & $-0 \cdot 019$ & $-6 \cdot 044 \pm 0.499$ & $-1 \cdot 338 \pm 0 \cdot 250$ & $-1 \cdot 25$ \\
\hline
\end{tabular}

${ }^{\mathrm{a}} \Delta G_{\text {red }}^{0}=\Delta H^{0}-T \Delta S_{\text {mol }}$

Table 4. Calculation of the absolute free energy of reduction $\left(\Delta G_{r e d}^{0}\right)$ and $E_{\text {red }}^{0}$ of Pheophytin- $a$ in DMF by DFTDPCM method. We have used 1 a.u. $=27 \cdot 2116 \mathrm{eV} .9$

\begin{tabular}{|c|c|c|c|c|c|c|}
\hline \multirow[b]{2}{*}{ Reaction } & \multicolumn{4}{|c|}{$(e V)$} & \multicolumn{2}{|c|}{ Mid-point $E_{\text {red }}^{0}(V)$} \\
\hline & $\triangle E_{D P C M}$ & $\Delta E_{\text {thermal }}$ & $-T \Delta S_{m o l}$ & $\Delta G_{r e d}^{0}{ }^{\mathrm{a}}$ & Calcd. & Obsd. \\
\hline Pheo(DMF) $+\mathrm{e}^{-}(\mathrm{g}) \rightarrow \mathrm{Pheo}^{-}(\mathrm{DMF})$ & $-3 \cdot 222 \pm 0 \cdot 173^{\mathrm{a}}$ & -0.079 & $-0 \cdot 027$ & $-3 \cdot 328 \pm 0 \cdot 173$ & $-1.032 \pm 0.173$ & $-0 \cdot 9$ \\
\hline Pheo(DMF) $+2 \mathrm{e}^{-}(\mathrm{g}) \rightarrow$ Pheo $^{2-}(\mathrm{DMF})$ & $-5.935 \pm 0 \cdot 173$ & $-0 \cdot 158$ & $-0 \cdot 019$ & $-6 \cdot 112 \pm 0 \cdot 173$ & $-1 \cdot 304 \pm 0 \cdot 173$ & $-1 \cdot 25$ \\
\hline
\end{tabular}

${ }^{\mathrm{a}} \Delta G_{\text {red }}^{0}=\Delta E_{D P C M}+\Delta E_{\text {thermal }}-T \Delta S_{\text {mol }}$

Table 5. Electrostatic contribution to the free energy of the system (i.e. pheophytin and its neighbors which are at a radial distance of $4 \AA$ from it) computed using FDPB solver employing the Delphi software. ${ }^{9}$

\begin{tabular}{lcc}
\hline Free energy of electrostatic interaction $\left(G_{e l}\right)$ in $e V$ & $\Delta G_{e l}(\mathrm{eV})$ \\
\hline Pheo- $a$ & Pheo- $a^{-}$ & \\
$-12 \cdot 080$ & -15.861 & -3.781 \\
\hline
\end{tabular}

level, were used for all the co-factors. The grid spacing was set to $0.29 \AA$, that is, there were 3.5 grid points per angstrom. The truncated model in figure 4 fills $70 \%$ of the grid box. Changing the grid size did not affect the result. A probe radius of $1.4 \AA$, which defines the water-accessible surface, was chosen. Periodic boundary conditions were calculated with the option 'coulombic' and the convergence criterion was chosen to be $0.001 \mathrm{kT} / \mathrm{e}$. Both linear and non-linear iterations were kept to a total of 1000 in number. The di-electric constant of the truncated model $\left(\varepsilon_{\text {in }}\right)$ was set to $4 \cdot 0,{ }^{20}$ and the dielectric constant of the solvent water $\left(\varepsilon_{\text {out }}\right)$ was set to $80 \cdot 0 .{ }^{21}$ This will give the electrostatic contribution towards the free energy change of the reduction reaction $\left(\Delta G_{e l}\right)$. The nonelectrostatic contribution $\left(\Delta G_{n o n-e l}\right)$ which comprises of the cavitation, dispersion and repulsion energy terms, can be obtained by employing the DPCM methodology at ROB3LYP/6-31G(d) level on the structure of Pheophytin- $a$ extracted from the pdb file with hydrogen atoms inserted and their positions optimized by $\mathrm{MM}+$. The same value of $G_{\text {non-el }}$ was obtained for both the neutral and charged species.
Table 6. Calculation of the absolute free energy of reduction $\left(\Delta G_{r e d}^{0}\right)$ and $E_{r e d}^{0}$ of pheophytin- $a$ within the membrane. $\Delta G_{\text {non-el }}$ was computed employing DPCM solvation method at ROB3LYP/6-31G(d) level on the pdb geometry. We have taken $\varepsilon_{\text {in }}=4 \cdot 0$ and $\varepsilon_{\text {out }}=80 \cdot 0 .{ }^{9}$

\begin{tabular}{|c|c|c|c|c|}
\hline \multicolumn{3}{|c|}{$(e V)$} & \multicolumn{2}{|c|}{ Mid-point $E_{\text {red }}^{0}(\mathrm{~V})$} \\
\hline$\Delta G_{e l}$ & $\Delta G_{\text {non-el }}$ & $\Delta G_{r e d}^{0}{ }^{\mathrm{a}}$ & Calcd. & Obsd. \\
\hline$-3 \cdot 781$ & $0 \cdot 0$ & $-3 \cdot 781$ & $-0 \cdot 58$ & -0.61 \\
\hline
\end{tabular}

Within the thylakoid membrane, the total free energy change of the one-electron reduction process $\left(\Delta G_{\mathrm{red}}^{0}\right)$ is shown in table 5 . The corresponding calculation of the reduction potential $\left(E_{\mathrm{red}}^{0}\right)$ is given in table 6.

The QM/MM/MC+Born/Onsager/Debye-Hückel method gave the one- and two-electron reduction potential values of Pheo- $a$ as $-0.92 \mathrm{~V}$ and $-1.34 \mathrm{~V}$ while the DFT-DPCM method gave the corresponding values as $-1.03 \mathrm{~V}$ and $-1.30 \mathrm{~V}$ respectively. These values are in excellent agreement with the experi- 
mentally reported values of $-0.9 \mathrm{~V}$ and $-1.25 \mathrm{~V}$ respectively. ${ }^{22}$ Furthermore, the FDPB method along with the DFT-DPCM technique gave the reduction potential value of Pheo- $a$ within the thylakoid membrane as $-0.58 \mathrm{~V}$. This is in close agreement with the experimentally reported value of $-0.61 \mathrm{~V}^{23}$ The change of the reduction potential from its value in vitro is attributable to the large electrostatic interaction with neighbouring polar species within the membrane.

In conclusion, theoretical evaluation of the redox properties of solvated species is a tedious process that requires an extremely detailed approach, especially since the molecule-medium interaction energy plays a major role in determining the redox behaviour. The reduction potentials are generally small quantities, of the order of $10^{-2}$ a.u., and a slight lack of accuracy in the calculation can result in a large error in the calculated number. An explicit accounting of the medium effects can give rise to an understanding of the variation of redox properties of the same species in different media.

\section{Acknowledgment}

$\mathrm{N}$ M gratefully acknowledges financial support from Council of Scientific and Industrial Research.

\section{References}

1. (a) Svensson B, Etchebest C, Tuffery P, van Kan P, Smith J and Styring S 1996 Biochemistry 36 14486; (b) Olsson M H M and Ryde U 1999 J. Biol. Inorg. Chem. 4 654; (c) Ryde U, Olsson M H M and Pierloot 2001 Theor. Comput. Chem. 9, 1; (d) Sigfridsson E, Olsson M H M and Ryde U 2001 J. Phys. Chem. B105 5546; (e) Torres R A, Lovell T, Noodleman L and Case D A 2003 J. Am. Chem. Soc. 125 1923; (f) Olsson M H M, Hong G and Warshel A $2003 \mathrm{~J}$. Am. Chem. Soc. 1255025

2. (a) Torres R A, Lovell T, Noodleman L and Case D A 2003 J. Am. Chem. Soc. 125 1923; (b) Noodleman L 1981 J. Chem. Phys. 75737

3. Olsson M H M, Hong G and Warshel A $2003 \mathrm{~J}$. Am. Chem. Soc. 1255025

4. (a) Corni S, Rienzo F D, Felice D E and Molinari E 2004 Theor. Biochem. Biophys. 102 328; (b) Zhang L $\mathrm{Y}$ and Friesner R A 1995 J. Phys. Chem. 99 16479; (c) Fajer J, Davis M S, Brune D C, Spaulding L, Borg
D and Forman A 1976 Brookhaven Symp. Biol. 28 74; (d) Davis M S, Forman A, Hanson L K and Fajer J 1979 J. Phys. Chem. 83 3325; (e) Heimdal J, Jensen K P, Devarajan A and Ryde U 2007 J. Biol. Inorg. Chem. 1249

5. (a) Kornyshev A, Newton M, Ulstrup J and Sanderson B 2005 Chem. Phys. 3191 (b) Newton M D 1984 Annu. Rev. Phys. Chem. 35437

6. (a) Parson W W and Warshel A 2004 Chem. Phys. 296 201; (b) Parson W W and Warshel A $2004 J$. Phys. Chem. B108 10474

7. (a) Datta S N and Priyadarshy S 1990 Chem. Phys. Lett. 173 360; (b) Mallik B and Datta S N 1994 Int. J. Quantum Chem. 52629 (c) Datta S N and Deshpande R 1997 Indian J. Pure Appl. Phys. 35 483; (d) Datta S N and Mallik B 1997 J. Phys. Chem. B101 4171; Datta S N, Nehra V and Jha A 1999 J. Chem. B103 8768; (e) Datta S N, Prabhakar B G S and Nehra V 1999 J. Phys. Chem. B103 2291; (f) Datta S N, Parendekar P V and Lochan R C 2001 J. Phys. Chem. B105 1442; (g) Datta S N, Sudhamsu J and Pandey A 2004 J. Phys. Chem. B108 8007; (h) Pandey A and Datta S N 2005 J. Phys. Chem. B109 9066

8. Datta S N and Mallik B 1995 Int. J. Quantum Chem. 53 37; Datta S N and Mallik B 1995 Int. J. Quantum Chem. 199761865

9. Datta S N and Mehta N J. Phys. Chem. B (in press)

10. Lewis A, Bumpus J A, Truhlar D G and Cramer C J 2004 J. Chem. Ed. 81596

11. Gaussian 03, Revision C. 02, Frisch M J et al 2004 Gaussian, Inc., Wallingford CT

12. Vreven T, Byun K S, Komáromi I, Dapprich S, Montgomery J A, Morokuma K and Frisch M J 2006 J. Chem. Theory Comput. 2815

13. Rappé A K, Casewit C J, Colwell K S, Goddard W A III and Skiff W M 1992 J. Am. Chem. Soc. 114 10024; Rappé A K and Goddard W A III $1991 \mathrm{~J}$. Phys. Chem. 953358

14. HyperChem Professional Release 7.01 for Windows, Hypercube Inc., Gainesville, FL 2002

15. Bockris J O M and Reddy A K N 1998 In Modern electrochemistry (New York and London: Plenum Press)

16. Rocchia W, Alexov E and Honig B 2001 J. Phys. Chem. B105 6507

17. Rocchia W, Sridharan S, Nicholls A, Alexov E, Chiabrera A and Honig B 2002 J. Comp. Chem. 23 128

18. Vriend G 1990 J. Mol. Graph. 852

19. Delphi V.4, release 1.0; Accelrys Inc.; Pisa, Italy, 2001

20. Ishikita H and Knapp E 2005 FEBS Lett. 5793190

21. Ishikita H and Knapp E 2005 JACS 1271963

22. Kazakova A A, Kisselev B A and Kozlov Y N 1989 Bioelectrochem. Bioenerget. 21367

23. Scheer H 1990 Chlorophylls (London: CRC) 